\title{
INTEGRAÇÃO DE INFORMAÇÕES NA AUTOMAÇÃO DE FORNOS INDUSTRIAIS COMBUSTOL*
}

Mario Teruo Enju ${ }^{1}$ Marcos Taccolini2

\section{Resumo}

Soluções flexíveis, com baixo custo de implantação e manutenção, de sistemas de controle de processos e informação utilizando tecnologias de ponta desenvolvidas em plataforma .Net Framework. Focadas inicialmente em área Metalúrgica, onde eficiência em custos e alta confiabilidade tem relevância fundamental para sua viabilização econômica, estas soluções podem também ser aplicadas em áreas Siderúrgica, Petroquímica e demais segmentos. Integração das informações do chão de fábrica com o sistema de gestão da empresa com objetivo de facilitar a tomada de decisões estratégicas. Banco de dados relacional é o principal repositório de dados para compartilhar informações com a rede corporativa, gerenciado por uma ferramenta de software em tempo real para as lógicas de negócios e camada de apresentação. Maior performance, facilidade de operação, disponibilidade de dados, OEE, manutenção simplificada, acesso e ações remotas são viabilizados, permitindo menor custo e maior eficiência.

Palavras-chave: Net framework; TI; Automação; Integração.

\section{INTEGRATION OF INFORMATION ON AUTOMATION OF COMBUSTOL} INDUSTRIAL OVENS

\section{Abstract}

Flexible solutions, with low installation and maintenance costs, to improve control and information systems using state-of-the-art technology developed in .Net framework. Focusing first in Metallurgic segment, where the goal is to combine low cost with high availability, providing the cost-benefit for, those solutions can be also be applied in other segments like Steel, Petrochemical and continuous process in general. The Integration of information from the plant floor to the management system facilitates strategic decisions. Relational databases are used as the main repository system to share the information across the company network, managed by a real-time Presentation and Business Layer software tool. Higher performance, easy-to-use operation, data availability, OEE information, reliable equipment's maintenance, remote access and actions are enabled, delivering cost reduction and higher efficiency.

Keywords: Net framework; IT; Process-control; HMI; Real-time supervisory. 


\section{INTRODUÇÃO}

\subsection{Visão Geral}

O objetivo de redução de custos e aumento de eficiência, mantendo a qualidade do controle e das operações em equipamentos é viabilizado mediante o uso de novas tecnologias de informação.

Atualmente, o mercado de automação e tecnologia da informação industrial disponibiliza ferramentas de controle e supervisão desenvolvidas com base em plataforma .Net framework, que utilizam de forma plena os sistemas operacionais mais atuais do mercado mundial, com ferramentas de programação de quarta geração (primeira geração, VMS; segunda geração: DOS; terceira: Windows; quarta: .NET)

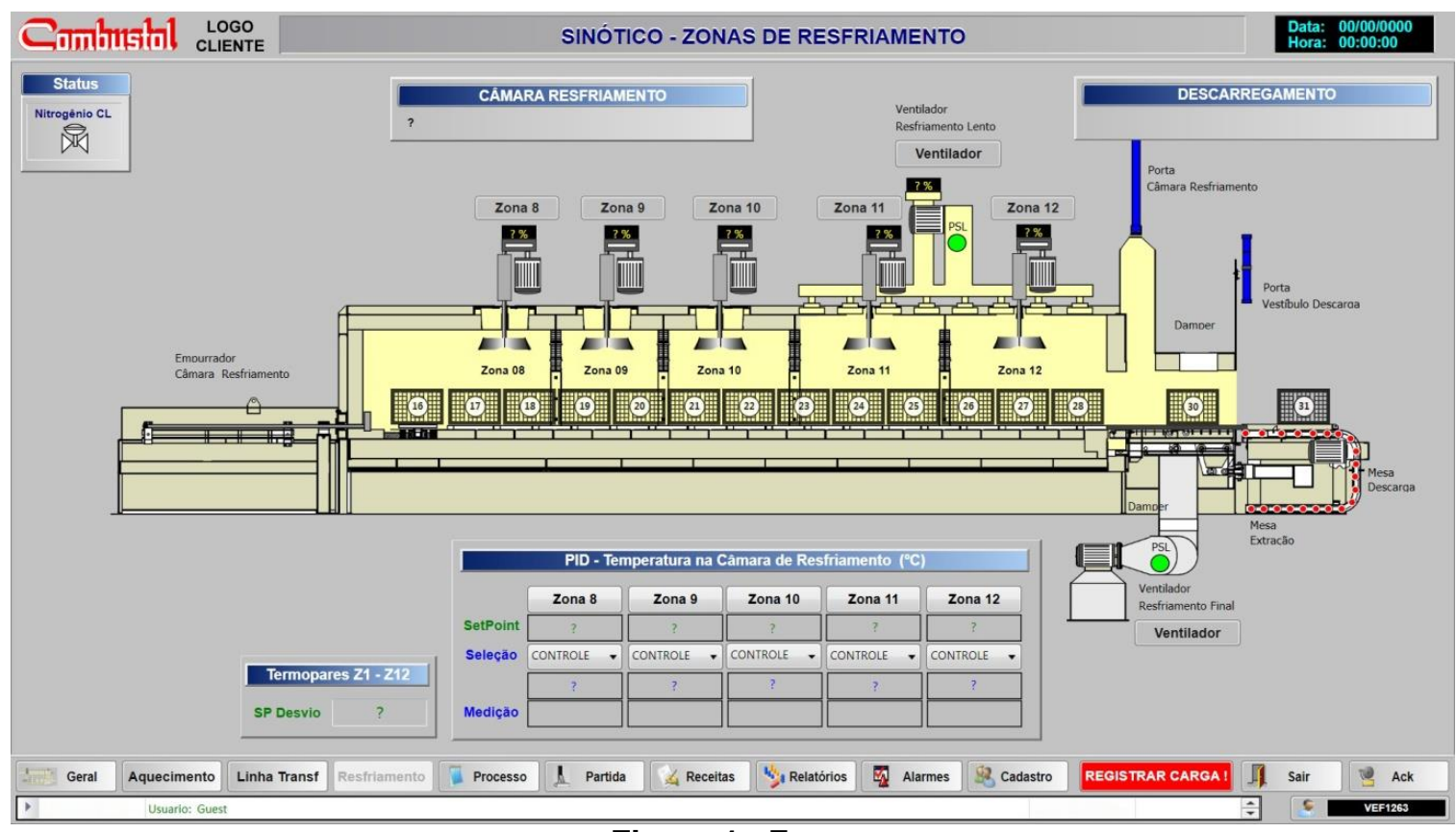

Figura 1. Forno

\subsection{Histórico}

A evolução tecnológica vem ocorrendo, nos últimos anos, com maior intensidade, principalmente quando falamos em sistemas de informática e plataformas computacionais.

Para cada atualização, muitas vezes o desenvolvimento feito em versões anteriores não são compatíveis com as novas versões, nem utiliza de forma adequada os recursos das novas plataformas operacionais, implicando em novo desenvolvimento de aplicativos, demandando custos de engenharia de desenvolvimento.

Geralmente, os custos incorridos em evoluções de plataformas (ex; Windows 95 para Windows XP para Windows 7) são significantes, impactando diretamente nos custos de equipamentos.

\subsection{Objetivo}

A Combustol, visando minimizar os custos de mão de obra especializada de desenvolvimento de software identificou que o melhor caminho é utilizar o conteúdo sistêmico já feito e reutilizá-lo independentemente de versão de plataforma ou até mesmo

* Contribuição técnica ao $18^{\circ}$ Seminário de Automação e TI Industrial, 23 a 26 de setembro de 2014, São Paulo, SP, Brasil. 
utilizando outras plataformas de sistema operacional. As ferramentas de software devem permitir o uso efetivo das novas plataformas operacionais, de forma transparente a engenharia de desenvolvimento.

\section{MATERIAL E MÉTODOS}

\section{$2.1 \quad$ Base}

A fim de sempre proporcionar a excelência na entrega de sistemas de gerenciamento e controle de equipamentos, a Combustol conta com a parceria de desenvolvedores de larga experiência na elaboração de minuciosos sistemas em tempo real, valorizando os produtos com a mais alta tecnologia em software.

Fornos Metalúrgicos são equipamentos, em sua maioria, propostos às empresas que necessitam manter baixos custos, porém necessitando de alta tecnologia pelo fato de envolver controle muito sofisticado e alta segurança de processo.

Controle e supervisão dos fornos são equipamentos controlados por PLC's de última geração, com interface de operação feita por supervisórios que atendem as necessidades requeridas.

O sistema supervisório atualmente utilizado pela Combustol foi criado por uma empresa nacional, especificamente para a plataforma Microsoft .NET Framework, que tem como característica, não carregar códigos legados do passado.

Com o uso da plataforma de software .NET Framework, o retrabalho necessário é mínimo quando ocorrem atualizações de plataforma de computadores e sistemas operacionais, possibilitando o desenvolvimento de meios para integração facilitada com banco de dados relacionais abertos, criando uma interface limpa para informes de dados de processo, assim como para receber diretrizes da engenharia de processo para o controle no chão de fábrica.

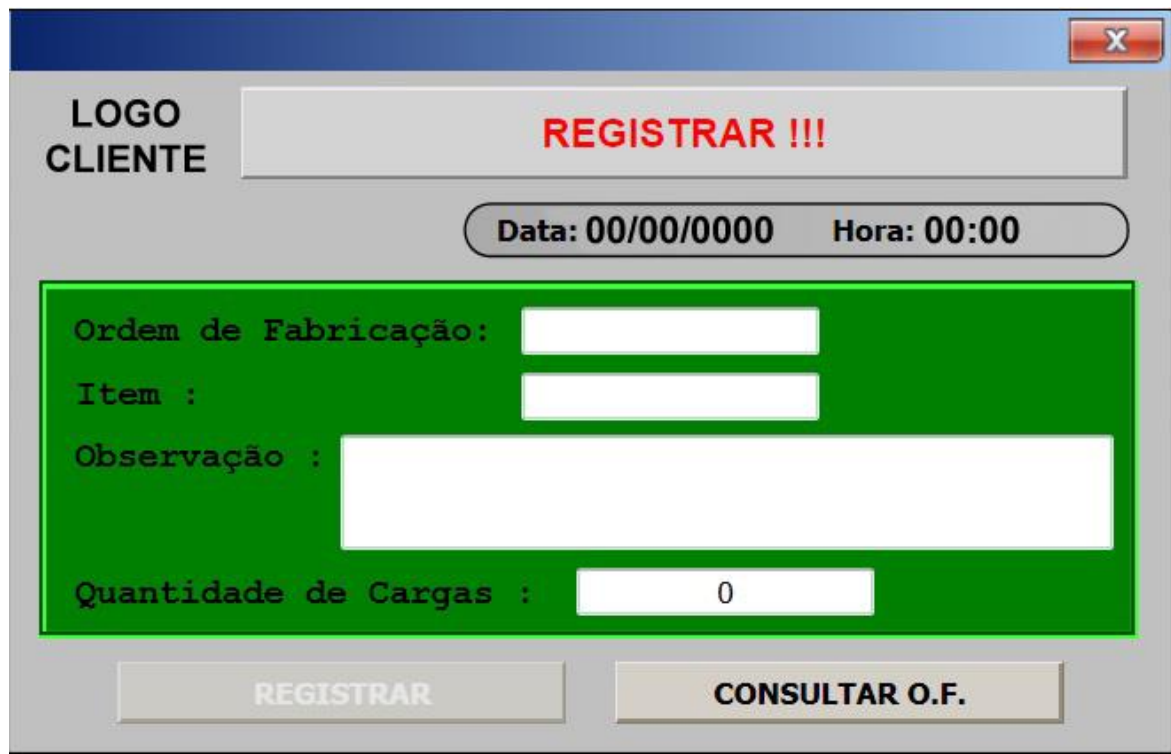

Figura 2. Integração com Banco de Dados

\subsection{Rastreabilidade de Carga}

Integrado a um banco de dados, o processo é visualizado de forma on-line atreladas às informações básicas requeridas e personalizadas.

* Contribuição técnica ao $18^{\circ}$ Seminário de Automação e TI Industrial, 23 a 26 de setembro de 2014, São Paulo, SP, Brasil. 


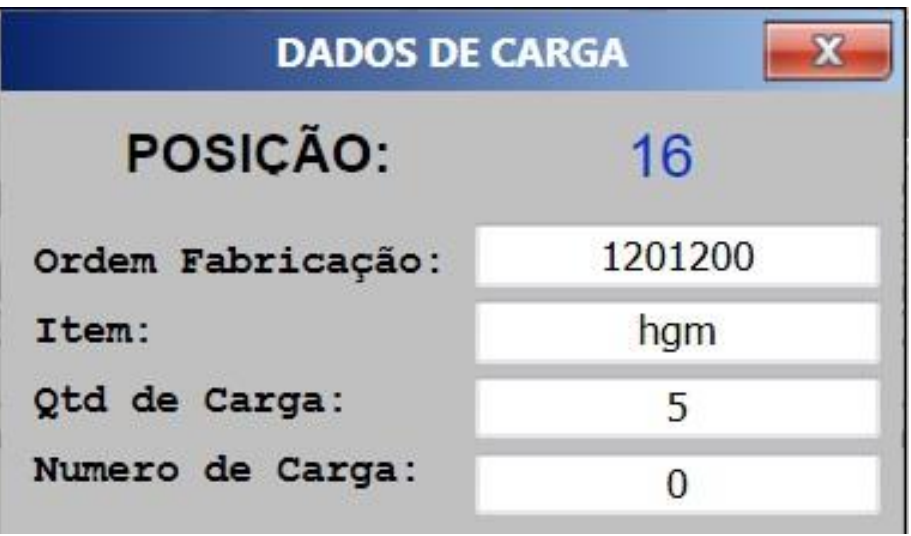

Figura 3. Identificação da carga na posição 16 no interior do Forno

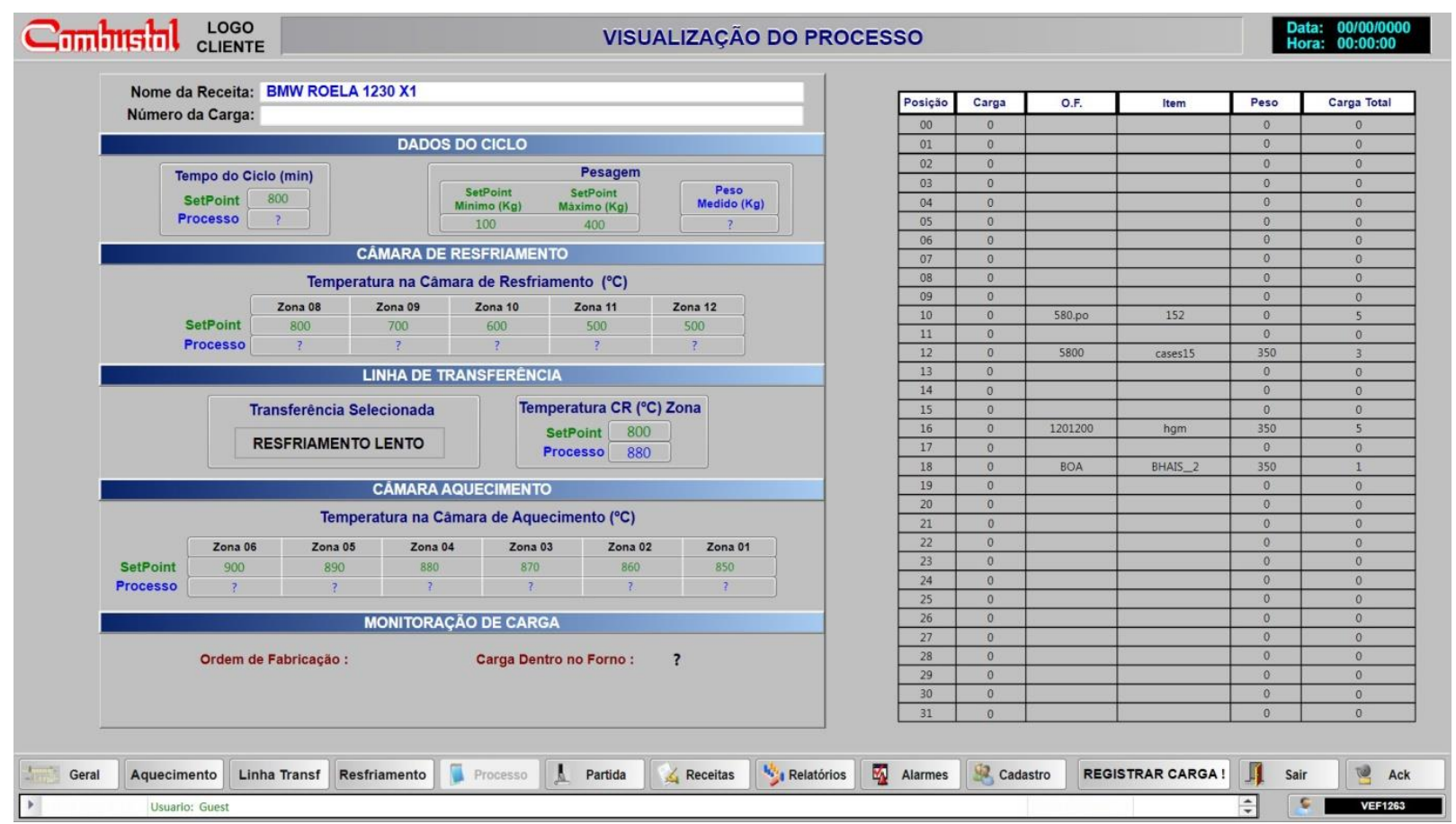

Figura 4. Vista geral de cargas em processo

\subsection{Back-up dos Dados de Processo}

De forma habitual, um computador adicional de back-up é fornecido para ficar interligado na rede do forno onde os dados históricos de processo são atualizados continuamente.

Desta forma, em caso de problemas no computador principal de controle, é feita a substituição pelo computador back-up que está com os dados de processo atualizados. Em sistemas críticos, este computador pode ser instalado com um par redundante do sistema principal, operando hot-stand-by, sem que haja necessidades de mudanças do projeto de software realizado pela engenharia.

\footnotetext{
* Contribuição técnica ao $18^{\circ}$ Seminário de Automação e TI Industrial, 23 a 26 de setembro de 2014, São Paulo, $S P$, Brasil.
} 


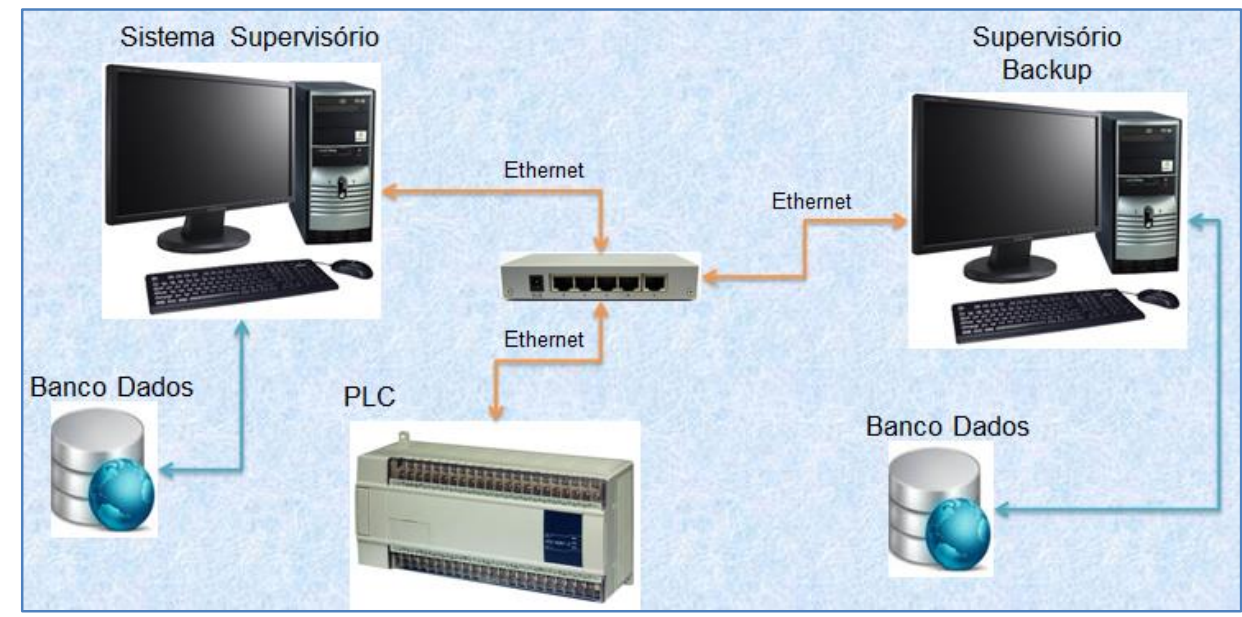

Figura 5. Arquitetura para backup dos dados

\subsection{Atendimento Remoto}

Equipamentos de controle instalados em clientes são preparados para que tenham acesso à internet, onde em situações de necessidade serão acessados remotamente pela Combustol.

Para o acesso remoto ao computador, atualmente utiliza-se o TeamViewer, um software simples, de fácil manipulação para "desktop remoto", que estabelece ligação com computadores independentemente da distância. Para acesso remoto aos dados de operação o sistema supervisório possui recursos nativos para telas remotas de operação.

\subsection{Arquitetura}

A arquitetura de software utiliza um gerenciador de tempo real e o sistema supervisório da Combustol, de acesso remoto, que atua como um centralizador e roteador dos dados em tempo real, permite que várias funções do sistema, aquisição de dados, visualização, cálculos, armazenamento, sejam desenvolvidas e atualizadas de forma independente.

O módulo de interface executa a leitura dos dados dos PLCs, e fornece setpoins e parâmetros de operação para o controlador.

O módulo de visualização fornece a interface local de operação e as mesmas interfaces de processo podem ser acessadas localmente em painéis touch-screen ou remotamente via web. O sistema também está preparado para o uso no futuro de dispositivos móveis acessados em tempo real aos dados do sistema.

O módulo de arquivamento e processamento implementa de forma simples e padronizada funções como gerenciamento de alarmes, histórico de dados e cálculos.

* Contribuição técnica ao $18^{\circ}$ Seminário de Automação e TI Industrial, 23 a 26 de setembro de 2014, São Paulo, $S P$, Brasil. 


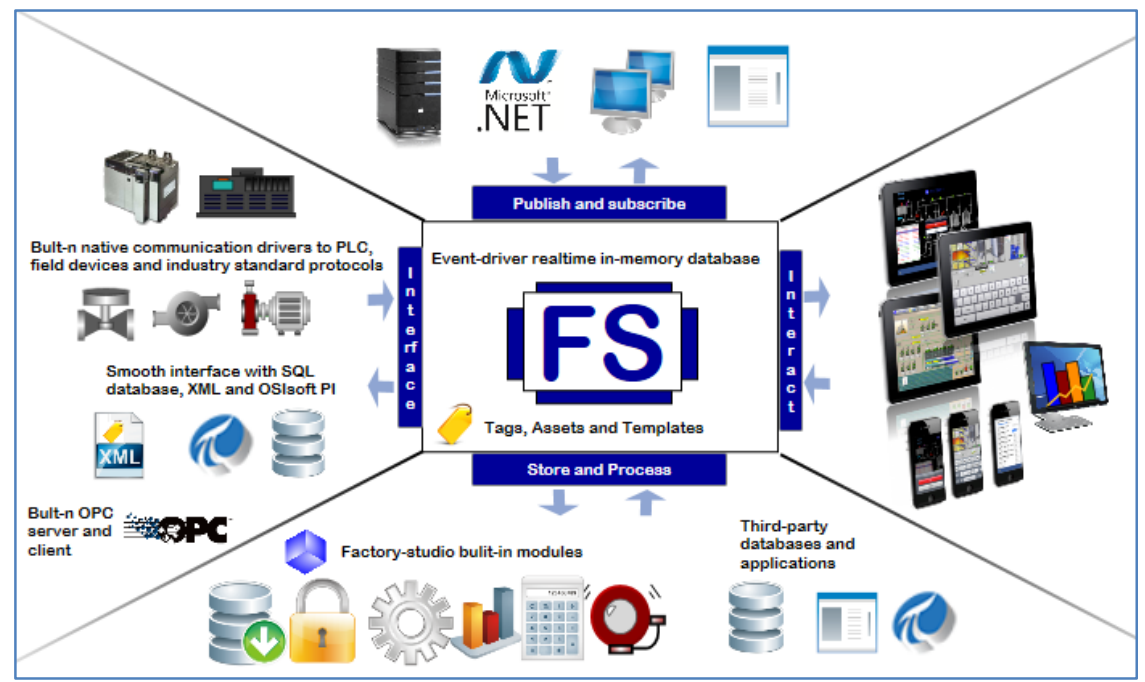

Figura 5. Modelo FactoryStudio

Finalmente, todos os dados relevantes do processo estão disponíveis para integração com a rede corporativa, utilizando bancos de dados SQL ou outros padrões de trocas de dados como arquivos XML ou web-services.

\section{RESULTADOS OBTIDOS/ ESPERADOS}

A solução da Combustol apresentada obtém a redução de custo na engenharia de desenvolvimento de software, onde as forças são direcionadas somente para aprimoramentos e aperfeiçoamentos das lógicas de controle e visualização, reduzindo os gastos em reescritas e adequações para diferentes plataformas.

Também garante a disponibilidade de informações de processo aos níveis gerenciais, a fim de facilitar e agilizar tomadas de decisões estratégicas.
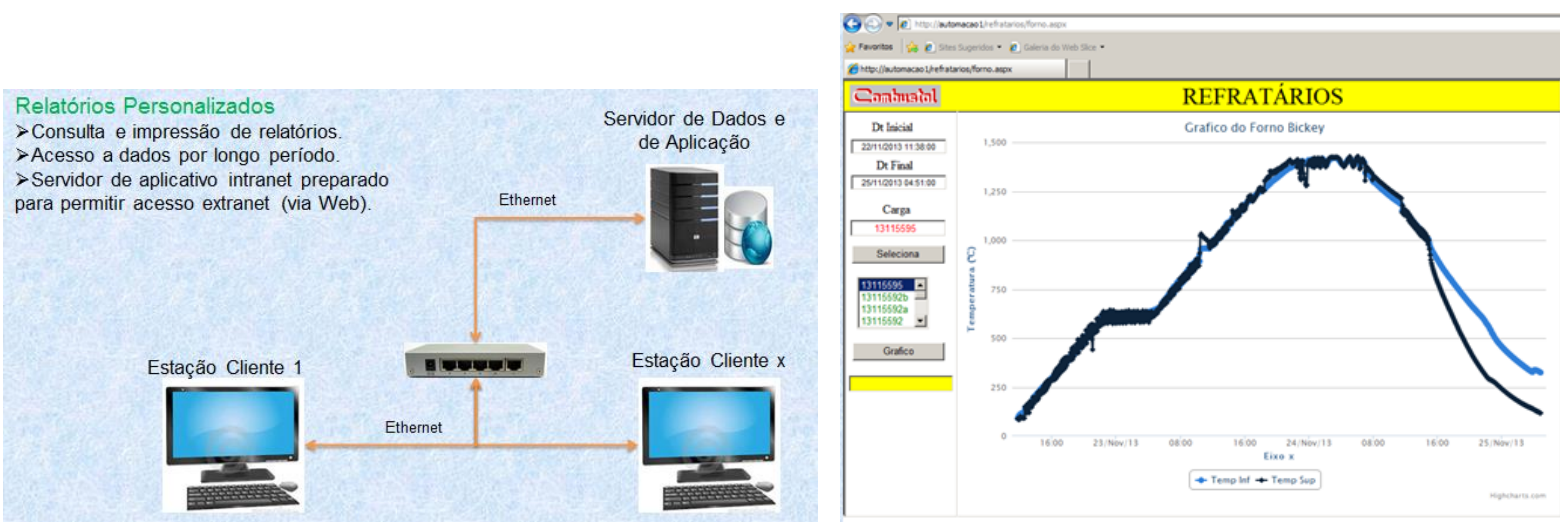

Figura 6. Disponibilidade de informações

As informações são distribuídas, de forma a evitar perda de informações, pois os dados não ficam armazenados nas células fabris (ambiente mais suscetível a problemas).

Consegue uma redução drástica no tempo de parada dos fornos com agilidade em atendimento através de acesso remoto. Problemas difíceis de identificação são monitorados continuamente via acesso remoto e viabilizado pelo baixo custo nessa modalidade, pois evitam custos de viagens, estadias e outros.

Além do mais, os atendimentos são acionados de forma preventiva e não mais, somente, de forma corretiva que implicava prejuízos para ambos os lados.

* Contribuição técnica ao $18^{\circ}$ Seminário de Automação e TI Industrial, 23 a 26 de setembro de 2014, São Paulo, $S P$, Brasil. 


\section{CONCLUSÃO}

Atualmente, o número de atendimentos a clientes, principalmente em localizações distantes da base da Combustol, em São Paulo, foi viabilizado e tornou-se frequente, trazendo com a solução, comodidade viabilidade e excelência em atendimentos de prevenção e melhoria contínua.

Para a Combustol, a disponibilidade técnica especializada de seus colaboradores tornou-se mais abrangente, já que possibilitou os atendimentos paralelos e é possível atender a toda a demanda durante os trajetos de locomoção dos colaboradores.

Finalmente, é possível afirmar que esta solução comercializada e aplicada pela Combustol traz melhorias quanto ao custo benefício, além de ser funcional e viável a todas as empresas que possuem equipamentos, sejam eles antigos ou de última geração.

\section{BIBLIOGRAFIA}

1 Especificação técnica dos Fornos Metalúrgicos de fabricação Combustol.

2 Descritivo funcional do sistema Tatsoft FactoryStudio

3 Taccolini, Marcos, "Gerações de Tecnologia em Automação Industrial”, August, 2011, Intech South America.

4 Vigiani, Roberto. "Tatsoft Distributed Technologies", December, 2010, Application Solutions Paper 